\title{
SOME UNIQUENESS THEOREMS ON RIEMANNIAN MANIFOLDS WITH BOUNDARY ${ }^{1}$
}

\author{
BY \\ Chuan-Chin Hsiung
}

\section{Introduction}

Let $M_{n}$ be a differentiable manifold of dimension $n$, and $X: M_{n} \rightarrow E_{n+m}$ a mapping of $M_{n}$ into a Euclidean space $E_{n+m}$ of dimension $n+m$ for any $m>0$. $M_{n}$, or rather $M_{n}$ together with the mapping $X$, is called an immersed submanifold of $E_{n+m}$ if the functional matrix of $X$ is of rank $n$ everywhere. The submanifold $M_{n}$ is said to be imbedded, if $X$ is one-one, that is, if $X(P)=X(Q), P, Q \in M_{n}$, implies that $P=Q$. In particular, when $m=1$, an immersed (imbedded) submanifold $M_{n}$ of the space $E_{n+m}$ is called an immersed (imbedded) hypersurface. Throughout this paper all manifolds are supposed to be of class $C^{3}$, and the dimension of a manifold $M_{n}$ is understood to be $n$.

Now let us consider an oriented immersed manifold $M_{n}$. Then to each point $P \in M_{n}$ there is a unique linear space $N$ of dimension $m$ normal to $X\left(M_{n}\right)$ at the point $X(P)$. For any unit normal vector $e_{r}(P)$ at the point $X(P)$ in the space $N$, we put

$$
I=d X \cdot d X, \quad I I_{r}=d e_{r} \cdot d X, \quad I I I_{r}=d e_{r} \cdot d e_{r},
$$

where $d X$ and $d e_{r}$ are vector-valued linear differential forms on $M_{n}$, and the dot denotes the scalar product of two vectors in the space $E_{n+m}$. The eigenvalues $k_{r 1}, \cdots, k_{r n}$ of $I I_{r}$ relative to $I$ are called the principal curvatures of the manifold $M_{n}$ associated with the unit normal vector $e_{r}(P)$. If the GaussKronecker curvature $K_{r}=k_{r 1} \cdots k_{r n}$ associated with the vector $e_{r}(P)$ is nonzero, the reciprocals $1 / k_{r 1}, \cdots, 1 / k_{r n}$, called the radii of principal curvatures associated with the vector $e_{r}(P)$, are the eigenvalues of $I I_{r}$ relative to $I I I_{r}$, which is also positive definite due to the assumption $K_{r} \neq 0$. In this case we introduce the $\alpha^{\text {th }}$ elementary symmetric function

$$
\left(\begin{array}{l}
n \\
\alpha
\end{array}\right) P_{r \alpha}=\sum 1 / k_{r 1} \cdots 1 / k_{r \alpha} \quad(1 \leqq \alpha \leqq n) .
$$

If $M_{n}$ is a hypersurface, then at each point $X(P)$ of $M_{n}$ there is only one unit normal vector $e_{r}$, and for $P_{r \alpha}$ associated with it we shall simply write $P_{\alpha}$.

Let $M_{n}$ be a closed oriented Riemannian manifold immersed in a Euclidean space $E_{n+m}$. By a normal frame $X e_{n+1} \cdots e_{n+m}$ on the manifold $M_{n}$ we mean a point $X$ of the manifold $M_{n}$ and an ordered set of mutually perpendicular unit vectors $e_{n+1}, \cdots, e_{n+m}$ normal to the manifold $M_{n}$ at the point

Received August 13, 1959.

1 This research was partially supported by the United States Air Force Office of Scientific Research of the Air Research and Development Command. 
$X . \quad M_{n}$ is called a star manifold, ${ }^{2}$ if there exist a point $O$, called a pole, in the manifold $M_{n}$ and a class $C^{2}$ field of normal frames $X e_{n+1} \cdots e_{n+m}$ over the manifold $M_{n}$ such that the Gauss-Kronecker curvature $K_{r}$ of the manifold $M_{n}$ and the support function $X \cdot e_{r}$ with respect to the pole $O$ are positive for every vector $e_{r}, n+1 \leqq r \leqq n+m$, at every point of the manifold $M_{n}$. This normal frame $X e_{n+1} \cdots e_{n+m}$ is called a fundamental normal frame of the star manifold $M_{n}$ at the point $X$. An $n$-dimensional star manifold with boundary is an $n$-dimensional compact subset of an $n$-dimensional star manifold. An $n$-dimensional convex hypersurface with boundary is an $n$-dimensional compact subset of the boundary of a convex region in an $(n+1)$ dimensional Euclidean space $E_{n+1}$, or is equivalently an $n$-dimensional compact subset of an $n$-dimensional imbedded hypersurface with positive GaussKronecker curvature everywhere. An $n$-dimensional convex hypercap is an $n$-dimensional convex hypersurface with boundary such that in the space $E_{n+1}$ there is at least one fixed direction, along which every line either is a tangent to the hypersurface or intersects the hypersurface at most at one point. It is obvious that a convex hypercap can never be closed.

Since Christoffel [5] established in 1865 his well-known uniqueness or rigidity theorem on closed convex surfaces in a space $E_{3}$, various uniqueness theorems of the same type on closed convex hypersurfaces have been obtained by different authors with different methods. It is natural to ask whether we can extend some of these uniqueness theorems on closed convex hypersurfaces to general immersed manifolds with boundary. In recent years the present and other authors have succeeded in deriving some new integral formulas, by means of which most classical uniqueness theorems can easily be extended to convex hypersurfaces with boundary satisfying a natural boundary condition. For uniqueness theorems on general immersed manifolds with boundary, due to the complication arising from the immersion, the only result we have so far is the generalization [9] of Christoffel's uniqueness theorem to two-dimensional immersed manifolds with boundary. The main purpose of the present paper is to further extend this uniqueness theorem to immersed manifolds of a general dimension $n>2$ with boundary, and to establish a uniqueness theorem on convex hypercaps by proving the following theorems.

Theorem I. Let $M_{n}$ and $M_{n}^{*}$ be two star manifolds, with boundaries $B_{n-1}$ and $B_{n-1}^{*}$ respectively, in a Euclidean space $E_{n+m}$ for any $m>0$. Suppose that there exists an orientation-preserving diffeomorphism $f$ of the manifold $M_{n}$ onto the manifold $M_{n}^{*}$ such that, at each pair of corresponding points, the manifolds $M_{n}$ and $M_{n}^{*}$ have a common fundamental normal frame $e_{n+1} \cdots e_{n+m}$ and equal $P_{r, n-1}$ defined by equation (1.2) and associated with each common unit normal vector $e_{r}, r=n+1, \cdots, n+m$. If the diffeomorphism $f$ restricted to the boundary $B_{n-1}$ is a translation (strictly speaking, is induced by a transla-

2 The author is indebted to the referee for his comment which leads to the definition of a star manifold in the present form. 
lation in the space $E_{n+m}$ ) carrying the boundary $B_{n-1}$ onto the boundary $B_{n-1}^{*}$, then the diffeomorphism $f$ is a translation carrying the whole manifold $M_{n}$ onto the whole manifold $M_{n}^{*}$.

THEOREM II. Let $M_{n}$ be a star manifold with a spherical boundary $B_{n-1}$ such that at every point $P_{r, n-2}^{\mu} P_{r, n-1}^{\nu}$ is constant for $\mu+\nu>0, \mu \geqq 0, \nu>0$ and for each vector $e_{r}$ of a fundamental normal frame of the manifold $M_{n}$. Then the manifold $M_{n}$ is a compact subset of an $n$-sphere.

TheOREM III. Let $M_{n}$ and $M_{n}^{*}$ be two oriented convex hypercaps with boundaries $B_{n-1}$ and $B_{n-1}^{*}$ respectively. Suppose that there exists an orientationpreserving diffeomorphism $f$ of the hypercap $M_{n}$ onto the hypercap $M_{n}^{*}$ such that at each pair of corresponding points the hypercaps $M_{n}$ and $M_{n}^{*}$ have the same outer normal vector and satisfy either

$$
P_{1} \leqq P_{1}^{*}, \quad P_{2} \geqq P_{2}^{*},
$$

or

$$
P_{1} \geqq P_{1}^{*}, \quad P_{2} \leqq P_{2}^{*},
$$

where $P_{\alpha}$ and $P_{\alpha}^{*}$ are defined by equation (1.2) for the hypercaps $M_{n}$ and $M_{n}^{*}$ respectively. If the diffeomorphism $f$ restricted to the boundary $B_{n-1}$ is a translation carrying the boundary $B_{n-1}$ onto the boundary $B_{n-1}^{*}$, then the diffeomorphism $f$ is a translation carrying the whole hypercap $M_{n}$ onto the whole hypercap $M_{n}^{*}$.

Corollary. Let $M_{n}$ be a convex hypercap with a spherical boundary $B_{n-1}$. If there is a constant $c$ such that, at each point of the hypercap $M_{n}$, either

$$
P_{1} \leqq c \leqq P_{2}^{1 / 2}
$$

or

$$
P_{1} \geqq c \geqq P_{2}^{1 / 2}
$$

then the hypercap $M_{n}$ is a compact subset of an n-dimensional hypersphere.

It should be noted that when $n=2$, the conditions (1.3) and (1.4) together are obviously weaker than the condition of Alexandroff [2], which can be stated as follows: At each pair of corresponding points the hypercaps $M_{2}$ and $M_{2}^{*}$ satisfy the condition $F\left(2 P_{1}, P_{2}\right)=F\left(2 P_{1}^{*}, P_{2}^{*}\right)$, where $F(U, V)$, for $U>0, U^{2} \geqq 4 V>0$, is a continuous function monotonely increasing in both variables $U$ and $V$. Furthermore, Grotemeyer [8] obtained Theorem III for $n=2$ in terms of the condition of Alexandroff, but actually only used the conditions (1.3) and (1.4) together in his proof.

\section{Immersed submanifolds in Euclidean space}

Suppose a Euclidean space $E_{n+m}$ is oriented. By a frame $X e_{1} \cdots e_{n+m}$ in the space $E_{n+m}$ we mean a point $X$ and an ordered set of mutually perpendicular unit vectors $e_{1}, \cdots, e_{n+m}$ with an orientation coherent with that of 
the space $E_{n+m}$ so that the determinant $\left|e_{1}, \cdots, e_{n+m}\right|$ is equal to +1 . To avoid confusion we shall use the following ranges of indices throughout this paper:

$$
\begin{gathered}
1 \leqq \alpha, \beta, \gamma \leqq n, \quad n+1 \leqq r, s, t \leqq n+m, \\
1 \leqq i, j, k \leqq n+m .
\end{gathered}
$$

Then we have

$$
e_{i} \cdot e_{j}=\delta_{i j}
$$

where $\delta_{i j}$ are the Kronecker deltas. Let $F(n, m)$ be the space of all frames in the space $E_{n+m}$, so that $\operatorname{dim} F(n, m)=\frac{1}{2}(n+m)(n+m+1)$. In $F(n, m)$ we introduce the linear differential forms $\omega_{i}^{\prime}, \omega_{i j}^{\prime}$ by the equations

$$
d X=\sum_{i} \omega_{i}^{\prime} e_{i}, \quad d e_{i}=\sum_{j} \omega_{i j}^{\prime} e_{j},
$$

where

$$
\omega_{i j}^{\prime}+\omega_{j i}^{\prime}=0 .
$$

Since $d(d X)=0$ and $d\left(d e_{i}\right)=0$, from equations (2.3) we have

$$
d \omega_{i}^{\prime}=\sum_{j} \omega_{j}^{\prime} \wedge \omega_{j i}^{\prime}, \quad d \omega_{i j}^{\prime}=\sum_{k} \omega_{i k}^{\prime} \wedge \omega_{k j}^{\prime}
$$

where $\wedge$ denotes the exterior product.

As explained in $\$ 1$, by an immersed submanifold in the space $E_{n+m}$ we mean an abstract manifold $M_{n}$ and a mapping $X: M_{n} \rightarrow E_{n+m}$ such that the induced mapping $X_{*}$ on the tangent space is univalent everywhere. Analytically, the mapping can be defined by a vector-valued function $X(P)$, $P \in M_{n}$. Our assumption implies that the differential $d X(P)$ of $X(P)$, which is a linear differential form on $M_{n}$ with value in $E_{n+m}$, has as values a linear combination of $n$, but not less than $n$, vectors $t_{1}, \cdots, t_{n}$. Since $X *$ is univalent, we can identify the tangent space of $M_{n}$ at the point $P$ with the vector space spanned by $t_{1}, \cdots, t_{n}$. A linear combination of the vectors $t_{1}, \cdots, t_{n}$ is called a tangent vector, and a vector perpendicular to them is called a normal vector. The immersion of $M_{n}$ in $E_{n+m}$ gives rise to a bundle $B$, whose bundle space is the subset of $M_{n} \times F(n, m)$ consisting of

$$
\left(P, X(P) e_{1} \cdots e_{n+1} \cdots e_{n+m}\right) \in M_{n} \times F(n, m)
$$

such that $e_{1}, \cdots, e_{n}$ are tangent vectors and $e_{n+1}, \cdots, e_{n+m}$ are normal vectors at the point $X(P)$.

Consider the inclusion mapping $\phi$ and the projection $p$ :

$$
B \stackrel{\phi}{\longrightarrow} M_{n} \times F(n, m) \stackrel{p}{\longrightarrow} F(n, m) .
$$

By putting

$$
\omega_{i}=(p \phi) \omega^{\prime}, \quad \omega_{i j}=(p \phi){ }^{*} \omega_{i j}^{\prime}
$$


from equations (2.4) and (2.5) we have

$$
\begin{gathered}
\omega_{i j}+\omega_{j i}=0 \\
d \omega_{i}=\sum_{j} \omega_{j} \wedge \omega_{j i}, \quad d \omega_{i j}=\sum_{k} \omega_{i k} \wedge \omega_{k j} .
\end{gathered}
$$

From the definition of the bundle $B$ it follows that $\omega_{r}=0$ and that $\omega_{\alpha}$ are linearly independent. Thus the first equation of (2.9) gives

from which we have

$$
\sum_{\alpha} \omega_{\alpha} \wedge \omega_{\alpha r}=0
$$

$$
\omega_{\alpha r}=\sum_{\beta} A_{r \alpha \beta} \omega_{\beta}, \quad A_{r \alpha \beta}=A_{r \beta \alpha} .
$$

If $\operatorname{det}\left(A_{r \alpha \beta}\right) \neq 0$ for some $r$, by introducing the matrix $\left(\lambda_{r \alpha \beta}\right)$ inverse to the matrix $-\left(A_{r \alpha \beta}\right)$ we have

$$
\omega_{\alpha}=\sum_{\beta} \lambda_{r \alpha \beta} \omega_{r \beta} .
$$

By means of equations (2.2), (2.3), (2.7), (2.10), and (2.11), equations (1.1) can be written as

$$
\begin{gathered}
I=\sum_{\alpha} \omega_{\alpha}^{2}, \quad I I I_{r}=\sum_{i} \omega_{r i}^{2} \\
I I_{r}=+\sum_{\alpha} \omega_{r \alpha} \omega_{\alpha}=-\sum_{\alpha, \beta} A_{r \alpha \beta} \omega_{\alpha} \omega_{\beta}=+\sum_{\alpha, \beta} \lambda_{r \alpha \beta} \omega_{r \alpha} \omega_{r \beta} .
\end{gathered}
$$

Suppose

$$
\operatorname{det}\left(\delta_{\alpha \beta}+\lambda_{r \alpha \beta} y\right)=\sum_{0 \leqq \gamma \leqq n}\left(\begin{array}{l}
n \\
\gamma
\end{array}\right) P_{r \gamma}\left(\lambda_{r}\right) y^{\gamma}
$$

where $y$ is a parameter. Then $P_{r \gamma}\left(\lambda_{r}\right)$ is a polynomial of degree $\gamma$ in $\lambda_{r \alpha \beta}$ for a fixed $r$, and it is easily seen that $P_{r \alpha}\left(\lambda_{r}\right)$ is equal to the invariant $P_{r \alpha}$ defined by equation (1.2).

Through a point in a Euclidean space $E_{n+m}$ let $A_{1}, \cdots, A_{n+m-1}$ be $n+m-1$ differentiable vector functions of $n$ variables $x^{1}, \cdots, x^{n}$, and let $J$ be any vector. Then the scalar product of the vector $J$ and the vector product $A_{1} \times \cdots \times A_{n+m-1}$ of the vectors $A_{1}, \cdots, A_{n+m-1}$ is given by

$$
J \cdot\left(A_{1} \times \cdots \times A_{n+m-1}\right)=(-1)^{n+m-1}\left|J, A_{1}, \cdots, A_{n+m-1}\right|,
$$

from which it follows that

$$
e_{1} \times \cdots \times \hat{e}_{r} \times \cdots \times e_{n+m}=(-1)^{n+m+r} e_{r},
$$

where the circumflex over $e_{r}$ indicates that the vector $e_{r}$ is to be deleted. In a previous paper of the author [10] we have combined the vector product of vectors and the exterior product of differentials to define the vector

$$
\begin{aligned}
& A_{1} \otimes \cdots \otimes A_{i-1} \otimes d A_{i} \otimes \cdots \otimes d A_{n+m-1} \\
& =\left(A_{1} \times \cdots \times A_{i-1} \times A_{i, \alpha_{i}} \times \cdots \times A_{n+m-1, \alpha_{n+m-1}}\right) d x^{\alpha_{i}} \\
& \wedge \cdots \wedge d x^{\alpha_{n+m-1}},
\end{aligned}
$$


where $i=1, \cdots, n+m-1$ and $A_{i, \alpha_{i}}=\partial A_{i} / \partial x^{\alpha_{i}}$. It is obvious that the vector (2.16) is independent of the order of the vectors $d A_{i}, \cdots$, $d A_{n+m-1}$. Let $d A$ be the area element of an immersed submanifold $M_{n}$ in the space $E_{n+m}$. Then by means of the combined operation $\otimes$ we obtain

$$
\begin{aligned}
& \underbrace{d X \otimes \cdots \otimes d X}_{n} \otimes e_{n+1} \otimes \cdots \otimes \hat{e}_{r} \otimes \cdots \otimes e_{n+m} \\
& =(-1)^{n+m+r} n ! e_{r} d A, \\
& \underbrace{d e_{r} \otimes \cdots \otimes d e_{r}}_{n} \otimes e_{n+1} \otimes \cdots \otimes \hat{e}_{r} \otimes \cdots \otimes e_{n+m} \\
& =(-1)^{n+m+r} n ! e_{r} K_{r} d A .
\end{aligned}
$$

From equations (2.3), (2.7), (2.15), (2.17), and (2.18) it follows that

$$
\begin{aligned}
d A & =\omega_{1} \wedge \cdots \wedge \omega_{n}, \\
K_{r} d A & =\omega_{r 1} \wedge \cdots \wedge \omega_{r n} .
\end{aligned}
$$

\section{Integral formulas for a pair of immersed manifolds with boundary}

Let $M$ be a compact differentiable manifold of dimension $n$ with boundary, and let $M_{n}$ and $M_{n}^{*}$ be immersed manifolds with boundaries $B_{n-1}$ and $B_{n-1}^{*}$ given by $X: M \rightarrow E_{n+m}$ and $X^{*}: M \rightarrow E_{n+m}$, respectively. Then $\$ 2$ can be applied to the manifolds $M_{n}$, and for the corresponding quantities and equations for the manifold $M_{n}^{*}$ we shall use the same symbols and numbers with a star respectively.

Suppose that there is a diffeomorphism $f$ of the manifold $M_{n}$ onto the manifold $M_{n}^{*}$ such that at each pair of corresponding points the manifolds $M_{n}$ and $M_{n}^{*}$ have parallel tangent spaces. Without loss of generality we may assume that

$$
e_{i}^{*}=e_{i} \quad(i=1, \cdots, n+m) .
$$

From equations $(2.3),(2.7),(2.3)^{*},(2.7)^{*}$, and (3.1) it follows that

$$
\omega_{r \alpha}^{*}=\omega_{r \alpha} .
$$

Now for the pair of immersed manifolds $M_{n}$ and $M_{n}^{*}$ we introduce the following differential forms:

$$
\begin{aligned}
& B_{\alpha, n-2-\alpha}=\sum_{r=n+1}^{n+m}(-1)^{r-1} \\
& \cdot|X, X^{*}, e_{n+1}, \cdots, \hat{e}_{r}, \cdots, e_{n+m}, d e_{r}, \underbrace{d X, \cdots, d X}_{\alpha}, \underbrace{d X^{*}, \cdots, d X^{*}}_{n-2-\alpha}|,
\end{aligned}
$$




$$
\begin{aligned}
& C_{r \beta, n-1-\beta}=(-1)^{m+r} \\
& \cdot|X, e_{n+1}, \cdots, \hat{e}_{r}, \cdots, e_{n+m}, d e_{r}, \underbrace{d X, \cdots, d X,}_{\beta} \underbrace{d X^{*}, \cdots, d X^{*}}_{n-1-\beta}|, \\
& C_{r \beta, n-1-\beta}^{*}=(-1)^{m+r} \\
& \quad \cdot|X^{*}, e_{n+1}, \cdots, \hat{e}_{r}, \cdots, e_{n+m}, d e_{r} \underbrace{d X, \cdots, d X}_{\beta}, \underbrace{d X^{*}, \cdots, d X^{*}}_{n-1-\beta}|, \\
& D_{r \beta, n-1-\beta}=(-1)^{m+r} \\
& \cdot \mid e_{r}, e_{n+1}, \cdots, e_{r-1}, e_{r+1}, \cdots, e_{n+m}, d e_{r}, \underbrace{d X, \cdots, d X, \underbrace{d X^{*}, \cdots, d X^{*}}_{n-1-\beta} \mid,}_{\beta}
\end{aligned}
$$

where $0 \leqq \alpha \leqq n-2$ and $0 \leqq \beta \leqq n-1$. By means of equation (2.14) and the operation $\otimes$ we obtain

$$
\begin{gathered}
C_{r \beta, n-1-\beta}=(-1)^{n+r-1} X \cdot E, \quad C_{r \beta, n-1-\beta}^{*}=(-1)^{n+r-1} X^{*} \cdot E \\
D_{r \beta, n-1-\beta}=(-1)^{n+r-1} e_{r} \cdot E
\end{gathered}
$$

where

$E=e_{n+1} \otimes \cdots \otimes \hat{e}_{r} \otimes \cdots \otimes e_{n+m} \otimes d e_{r}$

$$
\otimes \underbrace{d X \otimes \cdots \otimes d X}_{\beta} \otimes d \underbrace{X^{*} \otimes \cdots \otimes d X^{*}}_{n-1-\beta} * \text {. }
$$

From the definition of the operation $\otimes$ and the last equation of (3.7) it follows immediately that

$$
(-1)^{n+r-1} E=D_{r \beta, n-1-\beta} e_{r} .
$$

Thus the substitution of equation (3.8) in the first two equations of (3.7) gives

$$
C_{r \beta, n-1-\beta}=h_{r} D_{r \beta, n-1-\beta}, \quad C_{r \beta, n-1-\beta}^{*}=h_{r}^{*} D_{r \beta, n-1-\beta},
$$

where we have placed

$$
h_{r}=X \cdot e_{r}, \quad h_{r}^{*}=X^{*} \cdot e_{r} .
$$

By using equations (3.3), (3.4), (3.5), and (3.9), applying the ordinary rules for differentiation of determinants, and noticing the pairwise cancellation of terms, we can easily obtain

$$
\begin{aligned}
d B_{\alpha, n-2-\alpha} & =\sum_{\substack{n=n+1 \\
n+m}}\left(C_{r \alpha, n-1-\alpha}-C_{r, \alpha+1, n-2-\alpha}^{*}\right) \\
& =\sum_{r=n+1}^{n+m}\left(h_{r} D_{r \alpha, n-1-\alpha}-h_{r}^{*} D_{r, \alpha+1, n-2-\alpha}\right) \\
& (0 \leqq \alpha \leqq n-2) .
\end{aligned}
$$

Integrating both sides of equation (3.11) over the manifold $M_{n}$ and applying Stokes's theorem to the left side, we can arrive at the integral formulas 


$$
\int_{B_{n-1}} B_{\alpha, n-2-\alpha}=\int_{M_{n}} \sum_{r=n+1}^{n+m}\left(h_{r} D_{r \alpha, n-1-\alpha}-h_{r}^{*} D_{r, \alpha+1, n-2-\alpha}\right)
$$

$$
(0 \leqq \alpha \leqq n-2) .
$$

To apply the formulas (3.12) we introduce the differential forms of order $n$

$$
D_{r \alpha \beta}=(-1)^{m+r}
$$

$$
\begin{array}{r}
\cdot e_{r}, e_{n+1}, \cdots, e_{r-1}, e_{r+1}, \cdots, e_{n+m}, \underbrace{d e_{r}, \cdots, d e_{r}}_{n-(\alpha+\beta)}, \underbrace{d X, \cdots, d X}_{\alpha}, \underbrace{d X^{*}, \cdots, d X^{*}}_{\beta} \mid, \\
(0 \leqq \alpha, \beta \leqq n) .
\end{array}
$$

In virtue of equations (2.3), (2.7), (2.8), (2.11), (2.14), (2.15), (2.20), $(2.3)^{*},(2.7)^{*},(2.11)^{*},(3.2)$, and $(3.13)$ we can easily obtain, for two parameters $y$ and $y^{*}$,

$$
\begin{aligned}
& \sum_{0 \leqq \alpha+\beta \leqq n} \frac{n !}{\alpha ! \beta !(n-\alpha-\beta) !} y^{\alpha} y^{* \beta} D_{r \alpha \beta} \\
= & (-1)^{(n+1)(m-1)} \sum_{1 \leqq \alpha_{1}, \cdots, \alpha_{n} \leqq n} \varepsilon_{\alpha_{1} \cdots \alpha_{n}}\left(y \omega_{\alpha_{1}}+y^{*} \omega_{\alpha_{1}}^{*}+\omega_{r \alpha_{1}}\right) \\
& \wedge \cdots \wedge\left(y \omega_{\alpha_{n}}+y^{*} \omega_{\alpha_{n}}^{*}+\omega_{r \alpha_{n}}\right) \\
= & (-1)^{(n+1)(m-1)} n !\left(y \omega_{1}+y^{*} \omega_{1}^{*}+\omega_{r 1}\right) \wedge \cdots \wedge\left(y \omega_{n}+y^{*} \omega_{n}^{*}+\omega_{r n}\right) \\
= & (-1)^{(n+1)(m-1)} n ! \operatorname{det}\left(y \lambda_{r \alpha \beta}+y^{*} \lambda_{r \alpha \beta}^{*}+\delta_{\alpha \beta}\right) K_{r} d A,
\end{aligned}
$$

where $\varepsilon_{\alpha_{1} \cdots \alpha_{n}}$ is +1 or -1 according as $\alpha_{1}, \cdots, \alpha_{n}$ form an even or odd permutation of $1, \cdots, n$, and is zero otherwise. Now suppose

(3.15) $\operatorname{det}\left(y \lambda_{r \alpha \beta}+y^{*} \lambda_{r \alpha \beta}^{*}+\delta_{\alpha \beta}\right)=\sum_{0 \leqq \alpha+\beta \leqq n} \frac{n !}{\alpha ! \beta !(n-\alpha-\beta) !} y^{\alpha} y^{* \beta} P_{r \alpha \beta}$, so that $P_{r \alpha \beta}$ is a homogeneous polynomial of degrees $\alpha$ and $\beta$ in $\lambda_{r \rho \sigma}$ and $\lambda_{r \rho \sigma}^{*}(\rho, \sigma=1, \cdots, n)$ respectively. In particular, from equation (2.13) it follows that $P_{r \alpha 0}=P_{r \alpha}\left(\lambda_{r}\right)$. Comparison of equation (3.14) with equation (3.15) yields immediately

$$
D_{r \alpha \beta}=(-1)^{(n+1)(m-1)} n ! P_{r \alpha \beta} K_{r} d A \quad(0 \leqq \alpha, \beta \leqq n) .
$$

Substituting equation (3.16) in equation (3.12) we therefore obtain

$$
\begin{aligned}
& \int_{B_{n-1}} B_{\alpha, n-2-\alpha} \\
& =(-1)^{(n+1)(m-1)} n ! \int_{M_{n}} \sum_{r=n+1}^{n+m}\left(h_{r} P_{r \alpha, n-1-\alpha}-h_{r}^{*} P_{r, \alpha+1, n-2-\alpha}\right) K_{r} d A, \\
& \quad(0 \leqq \alpha \leqq n-2) .
\end{aligned}
$$

If the diffeomorphism $f$ of the manifold $M_{n}$ onto the manifold $M_{n}^{*}$ restricted to the boundary $B_{n-1}$ is a translation carrying the boundary $B_{n-1}$ onto the boundary $B_{n-1}^{*}$, then on the boundary $B_{n-1}, d X^{*}=d X$, and therefore 
$B_{0, n-2}=B_{n-2,0}$. From the two equations of (3.17), for which $\alpha=0$, $n-2$ respectively, we can easily obtain the integral formula

$$
\begin{aligned}
& 2 \int_{M_{n}} \sum_{r=n+1}^{n+m} h_{r}\left(P_{r 0, n-1}-P_{r, n-2,1}\right) K_{r} d A \\
& \quad=\int_{M_{n}} \sum_{r=n+1}^{n+m}\left[h_{r}^{*}\left(P_{r 1, n-2}-P_{r, n-1,0}\right)-h_{r}\left(P_{r, n-2,1}-P_{r 0, n-1}\right)\right] K_{r} d A .
\end{aligned}
$$

Addition of equation (3.18) to the one obtained by interchanging the roles of the two manifolds $M_{n}$ and $M_{n}^{*}$ in equation (3.18) thus gives

$$
\int_{M_{n}} \sum_{r=n+1}^{n+m}\left[h_{r}\left(P_{r 0, n-1}-P_{r, n-2,1}\right)+h_{r}^{*}\left(P_{r, n-1,0}-P_{r 1, n-2}\right)\right] K_{r} d A=0 .
$$

Let $F_{r}\left(u_{1}, \cdots, u_{n}\right), r=n+1, \cdots, n+m$, be $m$ functions in $n$ positive variables $u_{1}, \cdots, u_{n}$. We shall say that each function $F_{r}$ is of type $n-1$, if the following two conditions for each $r$ are satisfied:

(i) $F_{r}\left(P_{r 10}, \cdots, P_{r n 0}\right)=F_{r}\left(P_{r 01}, \cdots, P_{r 0 n}\right)$ implies that $P_{r, n-2,1} \geqq$ $P_{r 0, n-1}$, and therefore, by interchanging the two manifolds $M_{n}$ and $M_{n}^{*}$, that $P_{r 1, n-2} \geqq P_{r, n-1,0}$,

(ii) $F_{r}\left(P_{r 10}, \cdots, P_{r n 0}\right)=F_{r}\left(P_{r 01}, \cdots, P_{r 0 n}\right)$ and $P_{r, n-2,1}=P_{r 0, n-1}$ (or $P_{r 1, n-2}=P_{r, n-1,0}$ ) if and only if $\lambda_{r \alpha \beta}^{*}=\lambda_{r \alpha \beta}$ for $\alpha, \beta=1, \cdots, n$.

Theorem 3.1. Let $M_{n}$ and $M_{n}^{*}$ be two star manifolds, with boundaries $B_{n-1}$ and $B_{n-1}^{*}$ respectively, in a Euclidean space $E_{n+m}$ for any $m>0$, and let $F_{r}\left(u_{1}, \cdots, u_{n}\right), r=n+1, \cdots, n+m$, be $m$ functions of type $n-1$ in $n$ positive variables $u_{1}, \cdots, u_{n}$. Suppose that there exists an orientationpreserving diffeomorphism $f$ of the manifold $M_{n}$ onto the manifold $M_{n}^{*}$ such that, at each pair of corresponding points, the manifolds $M_{n}$ and $M_{n}^{*}$ have a common fundamental normal frame $e_{n+1} \cdots e_{n+m}$, and the functions $F_{r}\left(P_{r 10}, \cdots, P_{r n 0}\right)$ and $F_{r}\left(P_{r 01}, \cdots, P_{r 0 n}\right)$ have the same value for each $r$. If the diffeomorphism $f$ restricted to the boundary $B_{n-1}$ is a translation carrying the boundary $B_{n-1}$ onto the boundary $B_{n-1}^{*}$, then the diffeomorphism $f$ is a translation carrying the whole manifold $M_{n}$ onto the whole manifold $M_{n}^{*}$.

Proof. Applying a translation in the space $E_{n+m}$ if necessary, without loss of generality we may assume the poles in the star manifolds $M_{n}$ and $M_{n}^{*}$ to be coincident, so that $h_{r}>0$ and $h_{r}^{*}>0$ for $r=n+1, \cdots, n+m$ over the whole manifolds $M_{n}$ and $M_{n}^{*}$. Thus, due to the first property of the functions $F_{r}$ and the assumption that $K_{r}>0$, each term of the integrand of equation (3.19) is nonpositive, and equation (3.19) holds when and only when

$$
\begin{array}{r}
P_{r 0, n-1}=P_{r, n-2,1}, \quad P_{r, n-1,0}=P_{r 1, n-2} \\
\quad(r=n+1, \cdots, n+m) .
\end{array}
$$

By the second property of the functions $F_{r}$ we therefore obtain

$$
\lambda_{r \alpha \beta}^{*}=\lambda_{r \alpha \beta} \quad(\alpha, \beta=1, \cdots, n) .
$$


Substitution of equation (3.21) in equations (2.11) and (2.11)* gives immediately

$$
\omega_{\alpha}^{*}=\omega_{\alpha} \quad(\alpha=1, \cdots, n) .
$$

From equations $(3.22),(2.3),(2.7),(2.3)^{*}$, and $(2.7)^{*}$ it follows that $d X^{*}=$ $d X$ over the whole manifold $M_{n}$, and hence the proof of the theorem is complete.

In particular, if the second manifold $M_{n}^{*}$ in Theorem 3.1 is a compact subset of an $n$-sphere, then Theorem 3.1 becomes

TheOREM 3.2. Let $M_{n}$ be a star manifold with a spherical boundary $B_{n-1}$ in a Euclidean space $E_{n+m}$ for any $m>0$. If there are $m$ functions

$$
F_{r}\left(u_{1}, \cdots, u_{n}\right), \quad r=n+1, \cdots, n+m,
$$

in $n$ positive variables $u_{1}, \cdots, u_{n}$ with the following two properties for each vector $e_{r}$ of a fundamental normal frame at every point of the manifold $M_{n}$ :

(i) $F_{r}\left(P_{r 1}, \cdots, P_{r n}\right)=F_{r}\left(a, \cdots, a^{n}\right)=$ constant implies that $P_{r, n-2} \geqq a^{n-2}$,

(ii) $F_{r}\left(P_{r 1}, \cdots, P_{r n}\right)=F_{r}\left(a, \cdots, a^{n}\right)$ and $P_{r, n-2}=a^{n-2}$ imply that $\lambda_{r \alpha \beta}=a \delta_{\alpha \beta}$ for $\alpha, \beta=1, \cdots, n$,

then the manifold $M_{n}$ is a compact subset of an $n$-sphere of radius $a$.

For $m=1$, the integral formulas (3.12), (3.17), (3.18) and Theorems I, II, 3.1, 3.2 were obtained by Chern [3].

\section{Proofs of Theorems I and II}

Proof of Theorem $I$. Theorem I follows from Theorem 3.1 immediately if we can show that the $m$ functions $F_{r}=P_{r, n-1}, r=n+1, \cdots, n+m$, are of type $n-1$. To this end we need the following inequality of Garding [7]:

Let $P_{r, n-1}\left(\lambda_{r}^{(1)}, \cdots, \lambda_{r}^{(n-1)}\right)$ be the completely polarized form of the polynomial $P_{r, n-1}\left(\lambda_{r}\right)$ defined by equation $(2.13)$, so that

$$
P_{r, n-1}(\underbrace{\lambda_{r}, \cdots, \lambda_{r}}_{n-1})=P_{r, n-1}\left(\lambda_{r}\right), \quad P_{r, n-1}(\underbrace{\lambda_{r}, \cdots, \lambda_{r}}_{n-2}, \lambda_{r}^{*})=P_{r, n-2,1} .
$$

Then for positive definite symmetric matrices $\left(\lambda_{r \alpha \beta}^{(1)}\right), \cdots,\left(\lambda_{r \alpha \beta}^{(n-1)}\right)$ the following inequality is valid:

$$
P_{r, n-1}\left(\lambda_{r}^{(1)}, \cdots, \lambda_{r}^{(n-1)}\right) \geqq P_{r, n-1}\left(\lambda_{r}^{(1)}\right)^{1 /(n-1)} \cdots P_{r, n-1}\left(\lambda_{r}^{(n-1)}\right)^{1 /(n-1)},
$$

where the equality holds when and only when the $n-1$ matrices are pairwise proportional.

Suppose now $P_{r, n-1,0}=P_{r 0, n-1}$, which can be written as $P_{r, n-1}\left(\lambda_{r}\right)=$ 
$P_{r, n-1}\left(\lambda_{r}^{*}\right)$. Since $\left(\lambda_{r \alpha \beta}\right)$ and $\left(\lambda_{r \alpha \beta}^{*}\right)$ are positive definite, from the inequality (4.1) it follows that

$$
\begin{aligned}
P_{r, n-2,1}=P_{r, n-1}(\underbrace{\lambda_{r}, \cdots, \lambda_{r}}_{n-2}, \lambda_{r}^{*}) & \\
& \geqq P_{r, n-1}\left(\lambda_{r}\right)^{(n-2) /(n-1)} P_{r, n-1}\left(\lambda_{r}^{*}\right)^{1 /(n-1)}=P_{r, n-1}\left(\lambda_{r}^{*}\right),
\end{aligned}
$$

which is the first condition for the functions $P_{r, n-1}$ to be of type $n-1$. By interchanging the two manifolds $M_{n}$ and $M_{n}^{*}$ we have

$$
P_{r 1, n-2} \geqq P_{r, n-1}\left(\lambda_{r}\right) \text {. }
$$

The equality holds in (4.2) and (4.3) when and only when $\lambda_{r \alpha \beta}^{*}=\rho \lambda_{r \alpha \beta}$ for $\alpha, \beta=1, \cdots, n$. On the other hand, as in the proof of Theorem 3.1, by using equations (4.2), (4.3), and (3.19) it is easily seen that the equality holds in (4.2) and (4.3). Since $P_{r, n-1}\left(\lambda_{r}\right)=P_{r, n-1}\left(\lambda_{r}^{*}\right), \rho=1$, and therefore the second condition for the functions $P_{r, n-1}$ to be of type $n-1$ is satisfied.

Proof of Theorem II. By putting

$$
\lambda_{r \alpha \beta}^{(1)}=\cdots=\lambda_{r \alpha \beta}^{(n-2)}=\lambda_{r \alpha \beta}, \quad \lambda_{r \alpha \beta}^{(n-1)}=a \delta_{\alpha \beta} \quad(\alpha, \beta=1, \cdots, n),
$$

from inequality (4.1) we obtain

$$
P_{r, n-2}^{1 /(n-2)} \geqq P_{r, n-1}^{1 /(n-1)},
$$

where the equality holds when and only when $\lambda_{r \alpha \beta}=b \delta_{\alpha \beta}$ for $\alpha, \beta=1, \cdots, n$. Let $a>0$ be defined by

$$
P_{r, n-2}^{\mu} P_{r, n-1}^{\nu}=a^{\mu(n-2)+\nu(n-1)},
$$

so that

$$
P_{r, n-1}=a^{(\mu / \nu)((n-2)+n-1)} P_{r, n-2}^{-\mu / \nu} .
$$

From inequality (4.4) and equation (4.6) it follows that

$$
P_{r, n-2} \geqq P_{r, n-1}^{(n-2) /(n-1)}=a^{(n-2)((\mu / \nu)(n-2) /(n-1)+1)} P_{r, n-2}^{-(\mu / \nu)(n-2) /(n-1)},
$$

which implies $P_{r, n-2} \geqq a^{n-2}$, where the equality holds when and only when it holds in (4.4). Thus, if $P_{r, n-2}=a^{n-2}$, then $\lambda_{r \alpha \beta}=b \delta_{\alpha \beta}$ for $\alpha, \beta=1, \cdots, n$, and therefore $b=a$ in consequence of equation (4.5). Hence Theorem II follows from Theorem 3.2 by taking $F_{r}\left(P_{r 1}, \cdots, P_{r n}\right)=P_{r, n-2}^{\mu} P_{r, n-1}^{\nu}$.

\section{Integral formulas for convex hypercaps}

Let $M$ be a compact differentiable manifold of dimension $n$ with boundary; and let $M_{n}$ be a convex hypercap with boundary $B_{n-1}$, so that $M_{n}$ is an imbedded manifold given by $X: M \rightarrow E_{n+1}$ with positive Gauss-Kronecker curvature $K_{n+1}$ everywhere. Then $\S 2$ with $m=1$ can be applied.

In the space $E_{n+1}$, let $\xi$ be a fixed direction along which every line either 
is a tangent to the hypercap $M_{n}$ or intersects the hypercap $M_{n}$ at most at one point, so that by the definition of a convex hypercap we have

$$
\tau \equiv \xi \cdot e_{n+1} \geqq 0 \text {. }
$$

For the hypercap $M_{n}$ we introduce the following differential forms:

$$
\begin{aligned}
& A_{\alpha}=|\xi, X, \underbrace{d e_{n+1}, \cdots, d e_{n+1}}_{n-1-\alpha}, \underbrace{d X, \cdots, d X}_{\alpha}| \quad(0 \leqq \alpha \leqq n-1), \\
& D_{\beta}=|\xi, \underbrace{d e_{n+1}, \cdots, d e_{n+1}}, \underbrace{d X, \cdots, d X}| \quad(0 \leqq \beta \leqq n) .
\end{aligned}
$$

As in $§ 3$, by using exterior differentiation and Stokes's theorem we obtain

$$
\int_{M_{n}} D_{\alpha+1}=\int_{B_{n-1}} A_{\alpha} \quad(0 \leqq \alpha \leqq n-1) .
$$

Now let $M_{n}^{*}$ be another convex hypercap given by the imbedding $X^{*}: M \rightarrow E_{n+1}$, and suppose that there is a diffeomorphism $f$ of the hypercap $M_{n}$ onto the hypercap $M_{n}^{*}$ such that at each pair of corresponding points the hypercaps $M_{n}$ and $M_{n}^{*}$ have the same unit normal vector $e_{n+1}$. For this pair of hypercaps $M_{n}$ and $M_{n}^{*}$ we introduce the following differential forms:

$$
\begin{aligned}
& A_{\alpha \beta}=|\xi, X, \underbrace{d e_{n+1}, \cdots, d e_{n+1}}_{n-1-(\alpha+\beta)}, \underbrace{d X, \cdots, d X}_{\alpha}, \underbrace{d X^{*}, \cdots, d X^{*}}_{\beta}|, \\
& A_{\alpha \beta}^{*}=|\xi, X^{*}, \underbrace{d e_{n+1}, \cdots, d e_{n+1}}_{n-1-(\alpha+\beta)}, \underbrace{d X, \cdots, d X}_{\alpha}, \underbrace{d X^{*}, \cdots, d X^{*}}_{\beta}|, \\
& B_{\alpha \beta}=|X, X^{*}, \underbrace{d e_{n+1}, \cdots, d e_{n+1}}_{n-1-(\alpha+\beta)}, \underbrace{d X, \cdots, d X}_{\alpha}, \underbrace{d X^{*}, \cdots, d X^{*}}_{\beta}| \text {, } \\
& C_{\rho \sigma}=|X, \underbrace{d e_{n+1}, \cdots, d e_{n+1}}_{n-(\rho+\sigma)}, \underbrace{d X, \cdots, d X}_{\rho}, \underbrace{d X^{*}, \cdots, d X^{*}}_{\sigma}|, \\
& C_{\rho \sigma}^{*}=|X^{*}, \underbrace{d e_{n+1}, \cdots, d e_{n+1}}_{n-(\rho+\sigma)}, \underbrace{d X, \cdots, d X}_{\rho}, \underbrace{d X^{*}, \cdots, d X^{*}}_{\sigma}|, \\
& D_{\rho \sigma}=|\xi, \underbrace{d e_{n+1}, \cdots, d e_{n+1}}_{n-(\rho+\sigma)}, \underbrace{d X, \cdots, d X}_{\rho}, \underbrace{d X^{*}, \cdots, d X^{*}}_{\sigma}|,
\end{aligned}
$$

where $0 \leqq \alpha, \beta \leqq n-1$ and $0 \leqq \rho, \sigma \leqq n$. As in $\S 3$, it is easily seen that

$$
\tau C_{\rho \sigma}=h D_{\rho \sigma}, \quad \tau C_{\rho \sigma}^{*}=h^{*} D_{\rho \sigma} \quad(0 \leqq \rho, \sigma \leqq n),
$$

where we have placed

$$
h=X \cdot e_{n+1}, \quad h^{*}=X^{*} \cdot e_{n+1} .
$$


Exterior differentiation gives

$$
\begin{gathered}
d A_{\alpha \beta}=D_{\alpha+1, \beta}, \quad d A_{\alpha \beta}^{*}=D_{\alpha, \beta+1}, \\
d B_{\alpha \beta}=C_{\alpha, \beta+1}-C_{\alpha+1, \beta}^{*}=(1 / \tau)\left(h D_{\alpha, \beta+1}-h^{*} D_{\alpha+1, \beta}\right), \quad \text { if } \tau \neq 0 .
\end{gathered}
$$

Integrating both sides of each of equations (5.7) over the hypercap $M_{n}$ and applying Stokes's theorem to the left side we have

$$
\begin{array}{ll}
\int_{B_{n-1}} A_{\alpha \beta}=\int_{M_{n}} D_{\alpha+1, \beta}, \quad \int_{B_{n-1}} A_{\alpha \beta}^{*}=\int_{M_{n}} D_{\alpha, \beta+1}, & \\
\int_{B_{n-1}} B_{\alpha \beta}=\int_{M_{n}}(1 / \tau)\left(h D_{\alpha, \beta+1}-h^{*} D_{\alpha+1, \beta}\right), & \text { if } \tau \neq 0 .
\end{array}
$$

Essentially the same argument as that used in deriving equation (3.16) shows that

$$
D_{\alpha \beta}=(-1)^{n} n ! \tau P_{\alpha \beta} K_{n+1} d A \quad(0 \leqq \alpha, \beta \leqq n),
$$

where $P_{\alpha \beta}$ are defined, in terms of two parameters $y$ and $y^{*}$, by

$$
\begin{aligned}
\operatorname{det}\left(y \lambda_{n+1, \alpha \beta}+y^{*} \lambda_{n+1, \alpha \beta}^{*}+\right. & \left.\delta_{\alpha \beta}\right) \\
& =\sum_{0 \leqq \alpha+\beta \leqq n} \frac{n !}{\alpha ! \beta !(n-\alpha-\beta) !} y^{\alpha} y^{* \beta} P_{\alpha \beta} .
\end{aligned}
$$

We shall also write $P_{\alpha 0}=P_{\alpha}\left(\lambda_{n+1}\right)$ and $P_{0 \alpha}=P_{\alpha}\left(\lambda_{n+1}^{*}\right)$. Substituting equations (5.9) in equations (5.8) we thus arrive at the integral formulas

$$
\begin{aligned}
& \int_{B_{n-1}} A_{\alpha \beta}=(-1)^{n} n ! \int_{M_{n}} \tau P_{\alpha+1, \beta} K_{n+1} d A, \\
& \int_{B_{n-1}} A_{\alpha \beta}^{*}=(-1)^{n} n ! \int_{M_{n}} \tau P_{\alpha, \beta+1} K_{n+1} d A, \\
& \int_{B_{n-1}} B_{\alpha \beta}=(-1)^{n} n ! \int_{M_{n}}(1 / \tau)\left(h P_{\alpha, \beta+1}-h^{*} P_{\alpha+1, \beta}\right) K_{n+1} d A,
\end{aligned}
$$

if $\tau \neq 0$,

where $0 \leqq \alpha, \beta \leqq n-1$.

\section{Proof of Theorem III}

From equations (5.11) we can easily deduce different integral formulas, but for proving Theorem III we need only the following one:

$$
\begin{aligned}
\int_{B_{n-1}}\left(A_{10}-A_{01}+A_{01}^{*}\right. & \left.-A_{10}^{*}\right) \\
& =(-1)^{n} n ! \int_{M_{n}} \tau\left(P_{20}+P_{02}-2 P_{11}\right) K_{n+1} d A .
\end{aligned}
$$

From the assumption of Theorem III that the given diffeomorphism $f$ re- 
stricted to the boundary $B_{n-1}$ is a translation carrying the boundary $B_{n-1}$ onto the boundary $B_{n-1}^{*}$, it follows that over the boundary $B_{n-1}, d X^{*}=d X$, and therefore $A_{10}-A_{01}+A_{01}^{*}-A_{10}^{*}=0$. Thus the integral formula $(6.1)$ is reduced to

$$
\int_{M_{n}} \tau\left(P_{20}+P_{02}-2 P_{11}\right) K_{n+1} d A=0
$$

On the other hand, in a recent paper [4] we have established the

Lemma. If $\mu=\left(\mu_{\alpha \beta}\right)$ and $\mu^{*}=\left(\mu_{\alpha \beta}^{*}\right)$ are two positive definite symmetric matrices of order $n$ such that for a fixed $\gamma, 2 \leqq \gamma \leqq n$,

$$
P_{\gamma-1}(\mu) \leqq P_{\gamma-1}\left(\mu^{*}\right), \quad P_{\gamma}(\mu) \geqq P_{\gamma}\left(\mu^{*}\right),
$$

then

$$
Q_{\gamma}\left(\mu, \mu^{*}\right) \equiv P_{\gamma}(\mu)+P_{\gamma}\left(\mu^{*}\right)-2 P_{\gamma-1,1}\left(\mu, \mu^{*}\right) \leqq 0
$$

where the equality implies that $P_{\gamma}(\mu)=P_{\gamma}\left(\mu^{*}\right)$.

At each pair of corresponding points of the hypercaps $M_{n}$ and $M_{n}^{*}$ under the diffeomorphism $f$, we take the common unit outer normal vector to be the vector $e_{n+1}$; so that the matrices $\left(\lambda_{n+1, \alpha \beta}\right)$ and $\left(\lambda_{n+1, \alpha \beta}^{*}\right)$ are positive definite everywhere, and the conditions (1.3) or (1.4) are equivalent to the conditions (6.3) with $\gamma=2$. Since $Q_{2}\left(\mu, \mu^{*}\right)$ is symmetric with respect to the matrices $\mu$ and $\mu^{*}$, the above lemma gives

$$
P_{2}\left(\lambda_{n+1}\right)+P_{2}\left(\lambda_{n+1}^{*}\right)-2 P_{11}\left(\lambda_{n+1}, \lambda_{n+1}^{*}\right) \leqq 0,
$$

where the equality implies that $P_{2}\left(\lambda_{n+1}\right)=P_{2}\left(\lambda_{n+1}^{*}\right)$. From the inequalities (5.1), (6.5), and the assumption that $K_{n+1}>0$, it follows immediately that the integrand of equation (6.2) is nonpositive, and equation (6.2) holds when and only when the equality holds in (6.5). Thus

$$
P_{2}\left(\lambda_{n+1}\right)=P_{2}\left(\lambda_{n+1}^{*}\right),
$$

and hence Theorem III follows from the uniqueness theorem of AlexandroffFenchel-Jessen for convex hypersurfaces with boundary. ${ }^{3}$

\section{REFERENCES}

1. A. Alexandroff, Zur Theorie der gemischten Volumina von konvexen Körpern. II, Mat. Sbornik (N.S.), vol. 2 (1937), pp. 1205-1238 (Russian with German summary).

2. - - Sur les théorèmes d'unicité pour les surfaces fermées, C. R. (Doklady) Acad. Sci. URSS, vol. 22 (1939), pp. 99-102.

3. S. S. CHERN, Integral formulas for hypersurfaces in Euclidean space and their applications to uniqueness theorems, J. Math. Mech., vol. 8 (1959), pp. 947-955.

4. S. S. Chern, J. Hano and C. C. Hsiung, A uniqueness theorem on closed convex hypersurfaces in Euclidean space, J. Math. Mech., vol. 9 (1960), pp. 85-88.

${ }^{3}$ For the uniqueness theorem of Alexandroff-Fenchel-Jessen, see [1], [6] for closed convex hypersurfaces, and [3] for convex hypersurfaces with boundary. 
5. E. B. Christoffel, Ueber die Bestimmung der Gestalt einer krummen Oberfäche durch lokale Messungen auf derselben, J. Reine Angew. Math., vol. 64 (1865), pp. 193-209.

6. W. Fenchel and B. Jessen, Mengenfunktionen und konvexe Körper, Danske Vid. Selsk. Math.-Fys. Medd., vol. 16, no. 3, 1938.

7. L. G̊̊rding, An inequality for hyperbolic polynomials, J. Math. Mech., vol. 8 (1959), pp. 957-965.

8. K. P. Grotemeyer, Zur Flächentheorie im Grossen: Über die Abbildung durch parallele Normalen, Arch. Math., vol. 9 (1958), pp. 117-122.

9. C. C. HsIung, A uniqueness theorem on two-dimensional Riemannian manifolds with boundary, Michigan Math. J., vol. 5 (1958), pp. 25-30.

10. - Curvature and Betti numbers of compact Riemannian manifolds with boundary, Univ. e Politec. Torino, Rend. Sem. Mat., vol. 17 (1957-1958), pp. 95-131.

LEHIGH UNIVERSITY

Bethlehem, Pennsyluania 